\title{
MiR-298 Exacerbates Ischemia/Reperfusion Injury Following Ischemic Stroke by Targeting Act1
}

\author{
Hongxue Sun ${ }^{a} \quad$ Di Zhong $^{a} \quad$ Cheng Wang $^{b} \quad$ Yilei Sun ${ }^{a} \quad$ Jiaying Zhao ${ }^{c}$ \\ Guozhong Lia \\ aDepartment of Neurology, The First Affiliated Hospital, Harbin Medical University, Harbin, ${ }^{b}$ Department \\ of Environmental Hygiene, Public Health College, Harbin Medical University, Harbin, 'Department of \\ Thoracic Surgery, The Second Affiliated Hospital of Harbin Medical University, Harbin, China
}

\section{Key Words}

Microrna-298 • Nuclear factor activator 1 - Middle cerebral artery occlusion • Ischemic injury

\begin{abstract}
Background/Aims: This study investigated the role of the microRNA miR-298 and its target Act1 in ischemic stroke. Methods: Cell viability was assessed with the 3-(4,5-dimethythiazol-2yl)-2,5-diphenyl tetrazolium bromide assay. Apoptotic cells were detected by flow cytometry, and mRNA and protein expression were assessed by quantitative real-time PCR and western blotting, respectively. The regulatory relationship between miR-298 and Act1 was evaluated with the luciferase assay. To clarify the role of Act1 following ischemic stroke, the transcript was knocked down by short interfering RNA. The in vitro findings were validated in a mouse model of middle cerebral artery occlusion by administration of miR-298 mimic. Results: Act1 was upregulated whereas miR-298 was downregulated in ischemic stroke. miR-298 overexpression by transfection of a mimic suppressed Act1 protein levels in vitro and in vivo, and the luciferase assay showed that miR-298 directly binds to the $3^{\prime}$ untranslated region of the Act 1 transcript. miR-298 overexpression enhanced cell apoptosis and autophagy and exacerbated ischemic infarction and neurological deficits, effects that were exerted via negative regulation of Act1/ c-Jun N-terminal kinase (JNK)/nuclear factor (NF)-KB signaling and downstream autophagy pathways. Conclusions: Upregulation of miR-298 following ischemic stroke promotes brain injury in vitro and vivo by inhibiting the Act1/JNK/NF-KB signaling cascade and the downstream autophagy pathway. Therapeutic strategies that target miR-298 could be beneficial for the treatment of ischemic stroke.

\section{Introduction}

Stroke is a primary cause of disability and mortality worldwide [1], and is often treated by intravenous or intra-arterial thrombolysis [2]. Temporary cessation of blood flow to the 
brain and subsequent reperfusion can lead to irreversible tissue damage and cell death [3]. A major barrier to adequate treatment for ischemic injury is that drugs in the cerebrospinal fluid are prevented from reaching brain tissues by the blood-brain barrier [4].It becomes urgent to find out some new therapies to treat with the ischemic stroke and it is of great significance to understand the underlying mechanism.

Nuclear factor (NF)- $\kappa \mathrm{B}$ activator (Act) 1 activates both the c-Jun $\mathrm{N}$-terminal kinase (JNK) and canonical NF- $\kappa B$ pathways as an E3 ubiquitin ligase [5-7]. Both JNK and NF- $\kappa B$ signaling are involved in cell autophagy, but it is unclear whether Act1 is related to the autophagy pathway and cerebral ischemia-reperfusion. Micro (mi)RNAs are small, non-coding, singlestranded RNA molecules that negatively regulate target gene expression and thereby modulate various biological functions; they also influence the stability and translational efficiency of mRNAs. miRNAs play important roles in many diseases including cancer as well as heart and brain diseases, and may regulate factors involved in ischemia-reperfusion injury [8]. The miRNA (miR)-298 has been implicated in various human malignancies, including gastric, ovarian, and breast cancers [9-11], and a study showed that miR-298 may play a vital role in Alzheimer's disease [12]. In addition, our previous bioinformatics analysis revealed that Act1 is a potential target of miR-298. However, there have been no studies on the role of miR-298 in ischemic injury.

In this study, we investigated the role of miR-298 and Act1 in ischemic stroke and their mechanisms of action using in vitro oxygen glucose deprivation/reperfusion (OGD/R) and in vivo middle cerebral artery occlusion (MCAO) models. Our results indicate that miR-298, Act1, JNK, NF- $\kappa B$, and the downstream autophagy pathway can serve as therapeutic targets in the treatment of ischemic stroke.

\section{Materials and Methods}

The study including animal and cell experiments, and the experimental protocol is shown in Fig. 1.

\section{Cell lines and culture conditions}

N2a mouse neuroblastoma cells (Type Culture Collection of the Chinese Academy of Sciences, Shanghai, China) and human embryonic kidney (HEK)293T cells were grown in Dulbecco's modified Eagle's medium (DMEM) containing $10 \%$ fetal bovine serum, $100 \mathrm{U} / \mathrm{ml}$ penicillin G, and $100 \mu \mathrm{g} / \mathrm{ml}$ streptomycin at $37^{\circ} \mathrm{C}$ in a humidified atmosphere of $5 \% \mathrm{CO}_{2}$.

\section{OGD/R model}

$\mathrm{N} 2 \mathrm{a}$ cells were incubated in glucose-free DMEM and immediately transferred to a humidified atmosphere of $1 \% \mathrm{O}_{2}, 94 \% \mathrm{~N}_{2}$, and $5 \% \mathrm{CO}_{2}$ at $37^{\circ} \mathrm{C}$ for $1 \mathrm{~h}$. Reoxygenation for $12,24,48,72$, or $96 \mathrm{~h}$ was initiated by rapidly replacing the glucosefree DMEM with normal medium and culturing the cells under normal conditions. Control cell cultures were not deprived of oxygen and glucose and were cultured under normoxic conditions. For overexpression experiments, N2a cells were transfected with miR-298 mimic for $24 \mathrm{~h}$.

\section{Animals and surgery}

The study protocol was approved by the Institutional Animal Care and Use Committee of the First Affiliated Hospital of Harbin Medical University and was carried out in accordance with the Guide for the Care and Use of Laboratory Animals (National Research Council, 1996, USA).

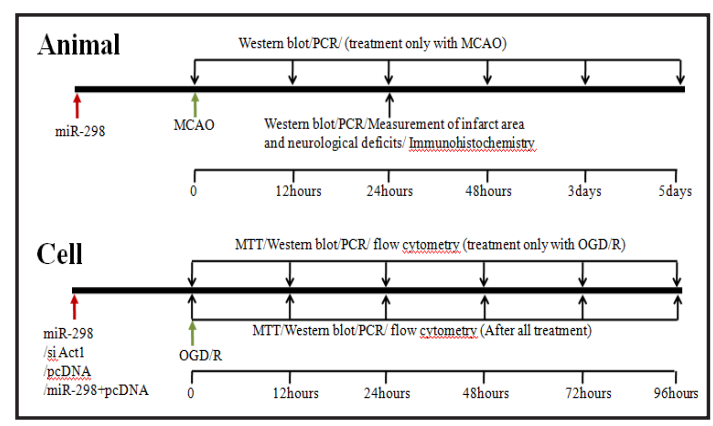

Fig. 1. Experimental timeline for miR-298, miR298, siAct1, pcDNA, miR-298+pcDNA, MCAO, OGD/R, MTT, Western blot, PCR, flow cytometry, measurement of infarct area and neurological deficits, and immunohistochemistry. 


\section{Cellular Physiology Cell Physiol Biochem 2018;48:528-539

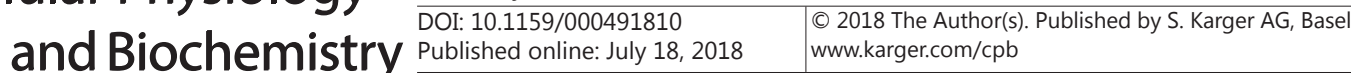

Sun et al.: Role of MiR-298 in Ischemia/Reperfusion Injury

Male C57BL/6 mice, 6-8 weeks old and weighing 20-25 g, (Beijing Vital River Laboratory Animal Technology Co., Beijing, China) were housed in individual cages on a 12:12-h light/dark cycle at $22^{\circ} \mathrm{C} \pm 2{ }^{\circ} \mathrm{C}$ with free access to food and water. The middle cerebral artery occlusion (MCAO) model of ischemic stroke was established as previously described $[13,14]$. Mice were deeply anesthetized by intraperitoneal injection of sodium pentobarbital $(40 \mathrm{mg} / \mathrm{kg}$ ). Blunt dissection was performed under a stereomicroscope (Stemi 2000; Carl Zeiss, Dresden, Germany) to expose the right common

Table 1. siRNAs targeting Act1

\begin{tabular}{lc}
\hline Construct & \multicolumn{1}{c}{ Sequence } \\
\hline Act1-63 & Forward: UAAGUCAGGGAAGCAUGAAdTdT \\
& Reverse: UUCAUGCUUCCCUGACUUAdTdT \\
Act1-64 & Forward: UCAGACCCAAGUUUACCUA dTdT \\
& Reverse: UAGGUAAACUUGGGUCUGAdTdT \\
Act1-65 & Forward: UCAGACAAAUCAGACCCAAdTdT \\
& Reverse: UUCAUGCUUCCCUGACUUAdTdT \\
\hline
\end{tabular}
carotid artery (CCA), external carotid artery (ECA), and internal carotid artery (ICA). The proximal end of the ipsilateral CCA was ligated, and an arterial clamp was applied to the CCA. This was followed by a small incision to the ECA artery between the permanent and temporary sutures and insertion of a Doccol suture $(0.21 \mathrm{~mm}$ in diameter $)$ into the ICA about $10 \mathrm{~mm}$ beyond the carotid bifurcation, which occluded the origin of the MCA. After $1 \mathrm{~h}$, the suture was removed and mice were allowed to recover for $12 \mathrm{~h}, 24 \mathrm{~h}, 48 \mathrm{~h}, 3$ days, and 5 days. Sham animals underwent the same procedure except for insertion of an intraluminal filament. The surgeries were carried out by the same technician. Rectal temperature was monitored during surgery and body temperature was maintained at $36^{\circ} \mathrm{C}\left( \pm 1^{\circ} \mathrm{C}\right)$ using a warming lamp.

\section{miRNA treatment for MCAO model}

Animals were anesthetized with 10\% chloral hydrate and placed in a stereotaxic apparatus (\#68001; RWD Life Science Co., Shenzen, China); a 3- $\mu$ l volume of $100 \mu \mathrm{M}$ miR-298 mimic (Genechem, Shanghai, China) was injected into the right cerebral ventricle over a 20 -min period. The stereotactic coordinates were as follows: anteroposterior, $0.3 \mathrm{~mm}$; mediolateral, $1.0 \mathrm{~mm}$; depth, $2.5 \mathrm{~mm}$. The injection of miR-298 or negative control mimic was considered successful if cerebral blood flow dropped to below baseline during injection. This blood flow rate was maintained for at least $20 \mathrm{~min}$. Mice were evaluated for neurologic deficits $24 \mathrm{~h}$ after reperfusion, and the brains were removed for analysis.

\section{Small interfering (si)RNA duplexes and Act1 knockdown}

SiRNA against Act1 were designed according to the Act1 gene sequence (GenBank Accession Number. NM_134000.3) (Table 1). siRNA against Act1 or control scrambled siRNA (Sigma-Aldrich) was transfected into N2a cells at a final concentration of 25 pmol using Lipofectamine 2000 (Invitrogen, Carlsbad, CA, USA) according to the manufacturer's instructions.

\section{Cell transfection}

Act1 cDNA lacking the 3' untranslated region (UTR) was inserted into the pcDNA3.1(+) vector (Invitrogen) to generate pcDNA3.1(+)-Act1. N2a cells were seeded in 96- or 6-well plates; when the cells reached $70 \%$ confluence, they were transfected with miR-298 mimic (100 nM) or pcDNA3.1(+)-Act1 using Lipofectamine 2000.

\section{Luciferase reporter assay}

The 3' UTR of the Act1 gene including miR-298-binding sites (wild type, Act1-3'UTR), as well as a mutant version (Act1-3'mUTR) were amplified by PCR and cloned into the pmirGLO vector (Promega, Madison, WI, USA) (Table 2). Correct insertion was confirmed by sequencing. For the luciferase reporter assay, HEK293T cells were seeded in a 24-well plate at $2 \times 10^{4} /$ well. When cells were $70 \%$ confluent, they were co-transfected with $100 \mathrm{ng}$ Act1-3'UTR or Act1-3'mUTR luciferase vector and $50 \mathrm{nM}$ miR-298 or negative control mimic. After $48 \mathrm{~h}$, cells were harvested and luciferase activity was evaluated with the Dual-Luciferase Reporter Assay System (Promega) according to the manufacturer's protocol. The assay was performed in triplicate. Each experiment was independently repeated at least three times.

\section{Determination of inhibition ratio}

The inhibition ratio for cell viability was determined with the 3-(4, 5-dimethythiazol- 2-yl)-2, 5-diphenyl tetrazolium bromide (MTT) assay. N2a cells were pretreated with miR-298 mimic or siAct1 or left untreated, and then subjected to OGD/R injury, which involved $1 \mathrm{~h}$ of OGD followed by 12, 24, 48, 72, or 


\section{Cellular Physiology Cell Physiol Biochem 2018;48:528-539 \begin{tabular}{l|l} 
and Biochemistry Published online: July 18, 2018 & $\begin{array}{l}\text { (c) 2018 The Author(s). Published by S. Karger AG, Basel } \\
\text { www.karger.com/cpb }\end{array}$
\end{tabular} \\ Sun et al.: Role of MiR-298 in Ischemia/Reperfusion Injury}

$96 \mathrm{~h}$ of reoxygenation. Cells were seeded in a 96-well plate at $1 \times$ $10^{5} / \mathrm{ml}$ in complete DMEM. After OGD/R, $20 \mu \mathrm{l}$ MTT were added to each well followed by incubation for $4 \mathrm{~h}$. The medium was removed and $150 \mu \mathrm{l}$ dimethyl sulfoxide was added to each well to dissolve the formazan crystals. Absorbance values were read on a spectrophotometer at a wavelength of $490 \mathrm{~nm}$. The number of cells was counted using a hemocytometer. The inhibition ratio of cell viability was calculated with the formula: Inhibition of cell viability $=(1-$ average absorbance value of the experimental group/average absorbance value of the control group) $\times 100 \%$. Results are presented as the average of a minimum of six wells.

Detection of apoptotic cells by flow cytometry

N2a cells were pretreated with miR-298 mimic or siAct1 or left untreated and then subjected to OGD/R (OGD for $1 \mathrm{~h}$ and reoxygenation for 12 , $24,48,72$, or $96 \mathrm{~h}$ ). After two washes with icecold phosphate-buffered saline, $100 \mu \mathrm{l}$ of the cell suspension were stained with $5 \mu \mathrm{l}$ annexin V-fluorescein isothiocyanate and $10 \mu \mathrm{l}$ propidium iodide (Sigma-Aldrich) followed by incubation for $15 \mathrm{~min}$ at room temperature in the dark. A 400- $\mu$ l volume of binding buffer was added to each sample followed by filtration through a 300-mesh nylon net and flow cytometry analysis on an EPICS XL instrument (Beckman Coulter, Brea, CA, USA). Data were analyzed with EXP032 ADC software (Beckman Coulter).

\section{Quantitative real-time ( $q R T$-) PCR}

Total RNA was extracted from N2a cells and reverse transcribed to cDNA using the Revert Aid First Strand cDNA Synthesis kit (Thermo Fisher Scientific, Beijing, China) according to the manufacturer's instructions on a CFX96 detection system (Bio-Rad, Hercules, CA, USA). qRT-PCR was carried out on a Plexor One-Step qRT-PCR system (Promega) using the primers listed in Table 3.

\section{Western blot analysis}

Total protein was extracted from mouse brain or from N2a cells and separated on a $10 \%$ polyacrylamide gel by sodium dodecyl sulfate polyacrylamide gel electrophoresis. The proteins were transferred to a polyvinylidene difluoride membrane (GE Healthcare, Little Chalfont, UK) that was blocked with $10 \%$ skimmed milk in Tween-20/Tris-buffered salt solution for $1 \mathrm{~h}$ at room temperature and then incubated overnight at $4^{\circ} \mathrm{C}$ with antibodies against the following proteins: Act1 (1:1000) and phosphorylated B cell lymphoma (pBcl)-2 (1:1000) (both from Santa Cruz Biotechnology, Santa Cruz, CA, USA); phosphorylated (p)JNK (1:2000), phosphorylated (p)NF- $\kappa B$ (1:500), and Caspase-3 (1:1000) (all from Cell Signaling Technologies, Danvers, MA, USA); and phosphorylated mammalian target of rapamycin (pmTOR) (1:1000) and Beclin1 (1:1000) (both from Chemicon International, Temecula, CA, USA). After incubating with horseradish peroxidase-conjugated goat anti-rabbit secondary antibody (Stressgen Biotechnologies, Victoria, BC, Canada), protein bands were visualized by chemiluminescence (GE Healthcare) and were quantitatively analyzed using the GelDoc-2000 imaging system (Bio-Rad). Glyceraldehyde 3-phosphate dehydrogenase was used as a loading control to normalize protein levels.

Immunohistochemistry

Paraffin-embedded tissues were deparaffinized, followed by rehydration and incubation with $10 \%$ hydrogen peroxide for $15 \mathrm{~min}$. Next, antigen retrieval was performed by heating the sections in citric acid 


\section{Cellular Physiology Cell Physiol Biochem 2018;48:528-539 and Biochemistry Published online: July 18, $2018 \quad \begin{aligned} & \text { DOI: 10.1159/000491810 } \\ & \begin{array}{l}\text { C) } 2018 \text { The Author(s). Published by S. Karger AG, Basel } \\ \text { www.karger.com/cpb }\end{array}\end{aligned}$}

Sun et al.: Role of MiR-298 in Ischemia/Reperfusion Injury

solution in a microwave for $1.5 \mathrm{~min}$. Sections were blocked using $5 \%$ goat serum for $30 \mathrm{~min}$ and incubated with antibodies against Beclin1, pBcl-2, pmTOR, pNF- $\kappa$ B, pJNK, and Caspase- 3 at $4^{\circ} \mathrm{C}$ overnight, followed by incubation with the appropriate secondary antibodies at $37^{\circ} \mathrm{C}$ for $30 \mathrm{~min}$. Finally, the sections were developed with diaminobenzidine and observed under a microscope.

Measurement of infarct area and neurological deficits

Brains removed from mice were frozen at $-20^{\circ} \mathrm{C}$ for $30 \mathrm{~min}$. Coronal brain sections were cut and stained with $2 \%$ 2, 3,5-triphenyltetrazolium chloride (TTC) solution (Sigma-Aldrich) at $37^{\circ} \mathrm{C}$ for $20 \mathrm{~min}$ followed by fixation in $4 \%$ formaldehyde for 1 day. Stained sections were imaged with a digital camera. To assess brain infarction 3 days after ischemia-reperfusion, mice were sacrificed by administering a lethal dose of sodium pentobarbital and were transcardially perfused. The brain was removed and coronal sections $(2 \mathrm{~mm}$ in thickness) were obtained using a mouse brain matrix and stained with TTC (Sigma-Aldrich). After the TTC staining, the software of Image J was used to calculate the infarct volume. Since each brain was sliced into six coronal sections ( $2 \mathrm{~mm}$ in thickness), the Image J was used to determine the infarct size of each section and then added up them together. After that, the total infarct size multiplied by the $2 \mathrm{~mm}$ thick to get the infarct volume of brain. Neurological impairment was scored as previously described $[15,16]$ at different time points on a blinded five-point Longa scale as follows: 0 , no neurological deficit; 1 , failure to fully extend left forepaw (mild focal neurological deficit); 2, circling to the left (moderate focal neurological deficit); 3 , falling to the left (severe focal deficit); and 4, exhibiting a depressed level of consciousness and unable to walk spontaneously.

\section{Statistical analysis}

Data were analyzed using SPSS v.13.01S software (Beijing Stats Data Mining Co., Beijing, China) and are presented as mean \pm standard deviation. Differences between means were evaluated with Student's t test or by one-way analysis of variance (ANOVA) (i.e., analysis of neurological deficits), and a two-way repeatedmeasures ANOVA was used for continuous variables (i.e., viability and apoptosis of N2a cells). $P$ values are two-tailed and $P<0.05$ was considered statistically significant.

\section{Results}

Act1 and miR-298 expression is altered by $O G D / R$ and MCAO

miR-298 expression was downregulated in N2a cells relative to the control group and decreased gradually over 12, 24, 48, 72, and $96 \mathrm{~h}$ after $1 \mathrm{~h}$ of OGD (Fig. 2A). Similarly, in MCAO mice, miR-298 level in the brain was decreased at 12 h, 24 h, 48 h, 3 days, and 5 days (Fig. 2B). In contrast, high levels of Act1 mRNA (Fig. 2C, D) and protein (Fig. 2E-H) were detected in both $\mathrm{OGD} / \mathrm{R} \mathrm{N} 2 \mathrm{a}$ cells and MCAO model mice relative to the respective control groups, with the expression increasing in a time-dependent manner. Thus, there was a reverse trending between the changes of Act1 and miR-298 expression levels.

\section{Act1 knockdown induces cell apoptosis and inhibits viability following $0 G D / R$}

To assess the role of Act1 in ischemic stroke, Act1 was silenced in OGD/R N2a cells by transfection of an siRNA against Act1 (Fig. 3A-C) and evaluating cell viability and apoptosis with the MTT assay and by flow cytometry, respectively (Fig. 3D). Compared to the control group, Act1 knockdown increased the rate of apoptosis and decreased viability (Fig. 3E). The protein level of Caspase- 3 was also detected by western blotting and was found to be increased after Act1 silencing (Fig. 3F, G). These data suggest that Act1 knockdown aggravates injury caused by OGD/R.

miR-298 overexpression induces apoptosis and inhibits viability following $0 G D / R$

To assess the role of miR-298 in ischemic stroke, OGD/R N2a cells were transfected with miR-298 mimic. The decrease in viability and increase in apoptosis of N2a cells after $1 \mathrm{~h}$ of OGD and $12,24,48,72$, and $96 \mathrm{~h}$ of reoxygenation was aggravated by miR-298 overexpression relative to the control group (Fig. 3E), and was accompanied by upregulation of Caspase-3 (Fig. 3F, G), suggesting that miR-298 induces apoptosis following ischemic stroke. 
Fig. 2. Changes in Act1 and miR-298 expression in OGD/R N2a cells and MCAO mice. A, B: Relative expression of miR-298 in OGD/R N2a cells (A) and MCAO mice (B). C-H: Relative Act1 mRNA (C, D) and protein $(\mathrm{E}-\mathrm{H})$ levels in $\mathrm{OGD} / \mathrm{R}$ N2a cells $(\mathrm{C}, \mathrm{E}, \mathrm{G})$ and MCAO mice (D, F, H). U6 was used as an internal control for quantification of miR-298 by qRT-PCR and glyceraldehyde 3-phosphate was used as a loading control for western blotting. $\mathrm{n}=10$ in each group. Statistical method: one-way ANOVA; * $\mathrm{P}<0.05$, ** $\mathrm{P}<0.01, * * * \mathrm{P}<0.001$ vs. control group.

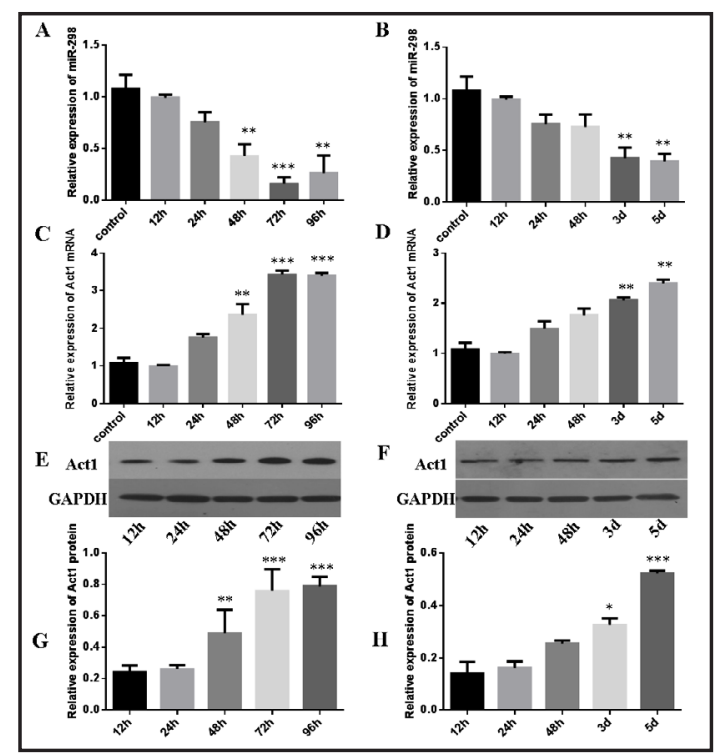

Fig. 3. Act1 knockdown has a similar effect as miR-298 overexpression. A-C: Efficiency of Act1 knockdown by different siRNAs. * $\mathrm{P}<0.05$ vs. normal group. D, E: Evaluation of cell viability with the MTT assay and detection of apoptotic cells by flow cytometry following transfection of miR-298 mimic or siRNA targeting Act1. Statistical method: one-way or two-way ANOVA; \# $\mathrm{P}<0.05$ vs. baseline; $* * * \quad \mathrm{P}<0.001$ vs. control group. F, G: Detection of Caspase-3 protein level by western blotting. * $\mathrm{P}<0.05$ vs. normal group.

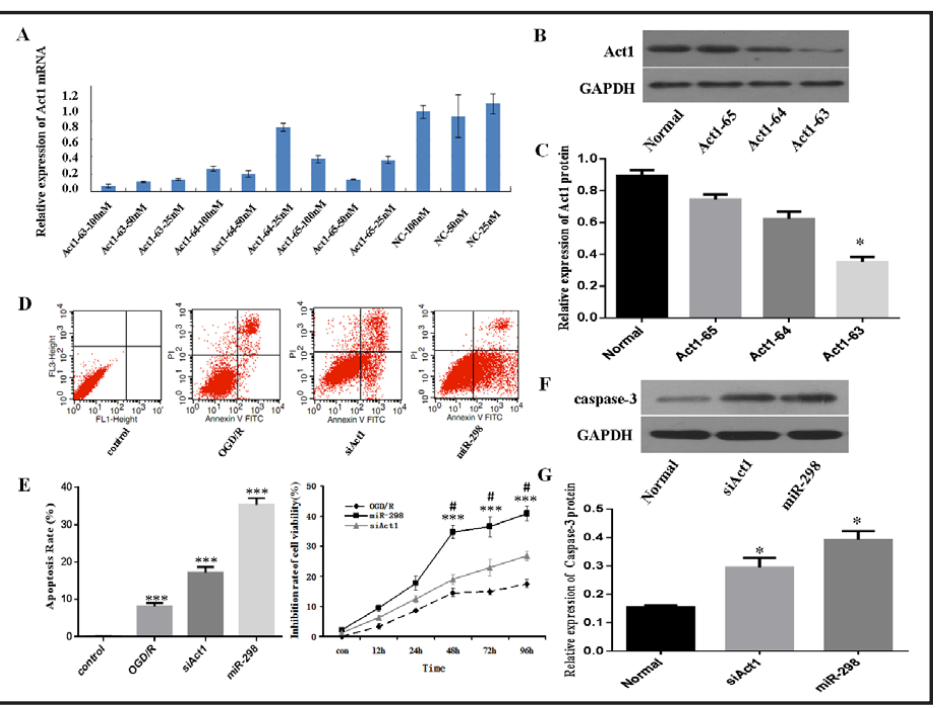

Act1 is a target gene of miR-298

A bioinformatics analysis identified Act1 is a potential target of miR-298 (Fig. 4A). We constructed reporter vectors harboring a wild-type or mutant miR-298-binding sequence to further investigate whether Act1 is directly targeted by miR-298. The vectors were cotransfected into $293 \mathrm{~T}$ cells along with negative control miRNA or miR-298 mimic. The results of the luciferase activity assay showed that miR-298 overexpression reduced luciferase activity in 293T cells co-transfected with the wild-type 3' UTR of Act1 (Fig. 4B). As expected, miR-298 mimic had no significant effect on the mutated reporter vectors.

To determine whether miR-298 regulates Act1 protein, we evaluated Act1 mRNA and protein levels in N2a cells subjected to $1 \mathrm{~h}$ OGD/24 h reoxygenation $48 \mathrm{~h}$ after transfection with miR-298 mimic by qRT-PCR and western blotting, respectively. Act1 mRNA and protein levels were both reduced upon transfection with miR-298 mimic as compared to controltransfected cells, in agreement with the results of the luciferase reporter assay (Fig. 4C-E). These results indicate that miR-298 negatively regulates Act1 by directly binding to the 3 ' UTR of the Act1 transcript. 
Fig. 4. Act1 is a direct target of miR-298. A: Act1 was identified as a potential target of miR-298 by bioinformatics analysis (RNAhybrid 2.2 database). B: miR-298 overexpression results in decreased luciferase expression in 293T cells co-transfected with the wild-type 3' UTR of Act1. C, D: Act1 mRNA and protein levels were decreased by transfection of miR-298 mimic as compared to the control. Statistical method: one-way ANOVA; ${ }^{*} \mathrm{P}<0.05$, ** $\mathrm{P}<0.01$, *** $\mathrm{P}<0.001$ vs. control group.

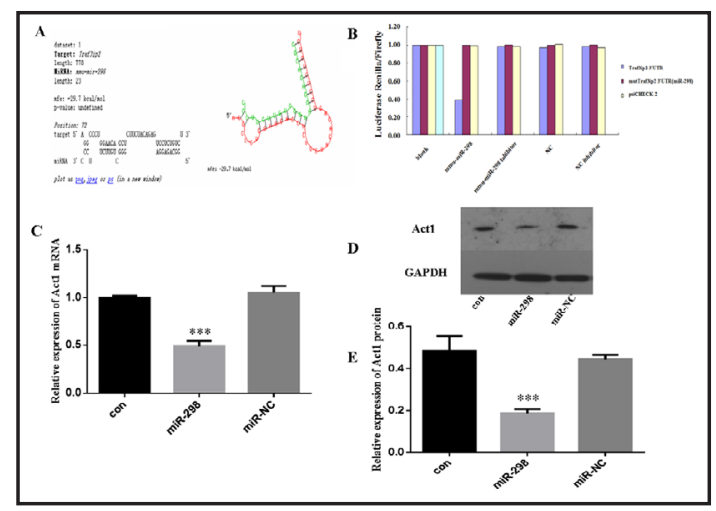

Fig. 5. Apoptosis is induced and cell viability was reduced by miR-298 overexpression in OGD/R N2a cells; these effects were reversed by Act1 knockdown. A, B: Act1 expression in N2a cells transfected with pcDNA3.1(+)-Act1. C-E: Evaluation of cell viability with the MTT assay and detection of apoptotic cells by flow cytometry following transfection of miR-298 mimic and pcDNA3.1(+)-Act1. Statistical method: t test, one-way or two-way ANOVA; ${ }^{\#} \mathrm{P}<0.05$ vs. baseline; ${ }^{*} \mathrm{P}<0.05$, *** $\mathrm{P}<0.001$ vs. control group.

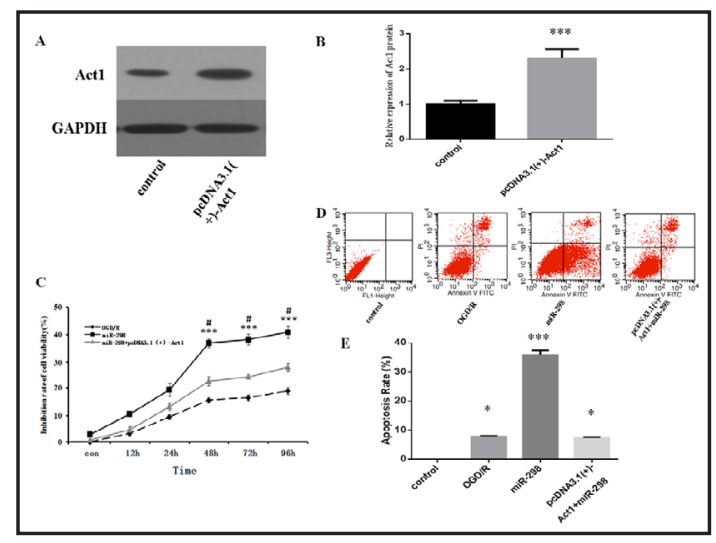

miR-298 induces apoptosis and reduces viability in OGD/R by targeting Act1

The viability of N2a cells decreased after $1 \mathrm{~h}$ of OGD and $24 \mathrm{~h}$ of reoxygenation; the same effect was observed by transfection of miR-298 mimic and Act1 knockdown (Fig. 3D, E). Furthermore, the rate of apoptosis and Caspase-3 protein level was increased in OGD/R N2a cells by miR-298 overexpression or Act1 knockdown (Fig. 3D-G). In a rescue experiment, we confirmed that Act1 overexpression in cells transfected with miR-298 mimic restored miR298-induced apoptosis and reduced cell viability (Fig. 5A-E).

miR-298 modulates autophagy-related proteins via Act1/NF- $\kappa B$ signaling in $O G D / R$

Since Act1 is an activator of NF- $\kappa B$ [5], we evaluated the contribution of miR-298 to the regulation of the Act1/NF- $\kappa \mathrm{B}$ pathway in N2a cells after OGD/R. A western blot analysis showed that pNF- $\kappa \mathrm{B}$ was upregulated in the OGD/R as compared to the normal group. Transfection of miR-298 mimic reduced the level of pNF- $\kappa \mathrm{B}$, which was restored by co-expression of miR-298 mimic and Act1. Additionally, transfection of miR-298 mimic decreased the level of the autophagy-related protein pmTOR, which was restored by cotransfecting Act1 (Fig. 6A). Act1 knockdown also resulted in downregulation of pNF-кB (Fig. $6 \mathrm{~B}, \mathrm{C}$ ) and downstream factors, as determined by qRT-PCR (Fig. 7A-F).

miR-298 induces autophagy-related proteins via Act1/JNK signaling following OGD/R

We examined whether Act1/JNK signaling and downstream factors are regulated by miR-298 following OGD/R N2a by western blot analysis. We found that pJNK was upregulated in the OGD/R as compared to the control group. miR-298 mimic increased the level of pJNK, an effect that was reversed by simultaneously overexpressing Act1. Transfection of miR-298 mimic also increased the levels of the autophagy-related proteins pBcl-2 and Beclin1, which were restored by co-transfecting pcDNA3.1(+)-Act1 (Fig. 6A). Act1 inhibition also resulted in upregulation of pJNK (Fig. 6B, C) and downstream factors, as determined by qRT-PCR (Fig. $8 \mathrm{~A}-\mathrm{F})$. 


\section{Cellular Physiology and Biochemistry

Fig. 6. Effects of miR-298 and Act1 overexpression and Act1 knockdown on protein levels of autophagyrelated genes and signaling components. A: Protein levels of Act1, Caspase-3, pNF- $\kappa$ B, pmTOR, pJNK, pBcl2, JNK, and Beclin1 in OGD/R N2a cells transfected with miR-298 mimic or pcDNA3.1(+)-Act1. B, $\mathrm{C}$ : Protein levels of $\mathrm{pNF}-\kappa \mathrm{B}$ and $\mathrm{pJNK}$ following transfection with miR-298 mimic or siRNA targeting Act1. Statistical method: one-way ANOVA; * $\mathrm{P}<0.05$ vs. normal group.

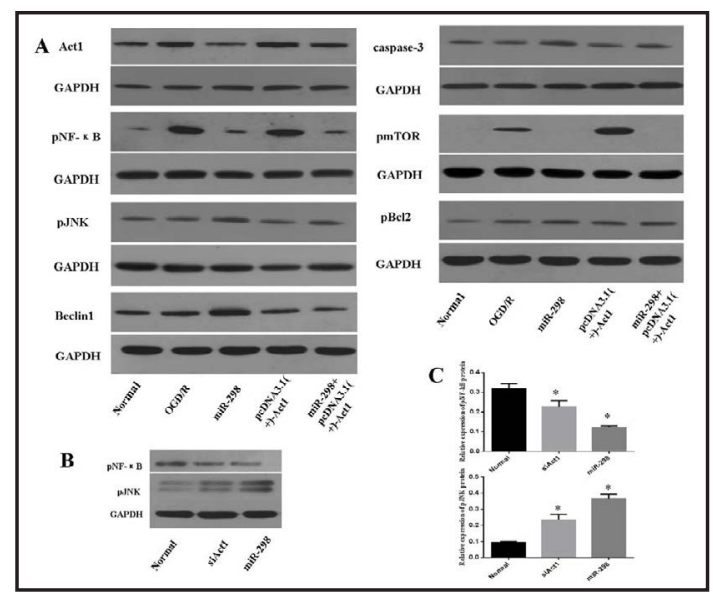

Fig. 7. Relative mRNA and protein levels of apoptosis and autophagyrelated genes in OGD/R N2a cells. A, C, E, G: Protein levels of Act1, Caspase-3, pNF- $\mathrm{B}$, pmTOR. B, D, F, $\mathrm{H}$ : mRNA levels of Act1, Caspase-3, NF- $\kappa B$, and mTOR. Statistical method: one-way ANOVA; *P<0.05, ${ }^{* *} \mathrm{P}<0.01,{ }^{* * *} \mathrm{P}<0.001$ vs. normal group.

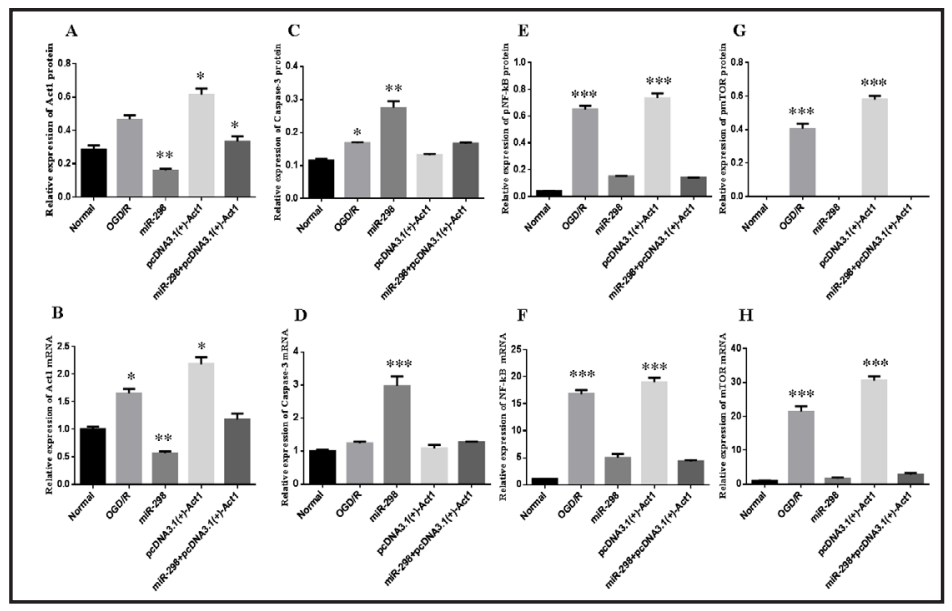

Fig. 8. Relative mRNA and protein levels of autophagy-related genes and signaling components in OGD/R N2a cells. A, C, E: Protein levels of pJNK, pBcl-2, and Beclin1. B, D, F: mRNA levels of JNK, Bcl2, and Beclin1. Statistical method: one-way ANOVA; * $\mathrm{P}<0.05,{ }^{* *} \mathrm{P}<0.01,{ }^{* * *} \mathrm{P}<0.001$ vs. normal group.

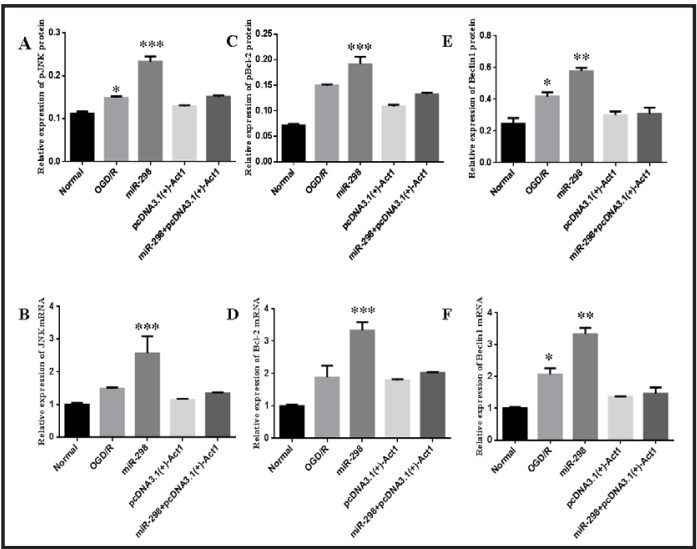

Downregulation of miR-298 is associated with activation of Act1/JNK/NF- $\kappa B$ signaling in vivo

To investigate the role of Act1 and miR-298 in ischemic/reperfusion injury in vivo, we examined the expression of Act1 in a mouse model of MCAO by western blotting. The results showed a similar trend to that observed in OGD/R N2a cells (Fig. 2E-H). The gradual decrease in miR-298 following MCAO (Fig. 2B) was associated with high levels of pJNK, pNF-кB, pmTOR, Beclin1, pBcl-2, and Caspase-3 in the brain, as determined by immunohistochemistry (Fig. 9). 


\section{Cellular Physiology

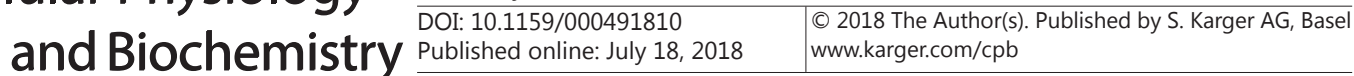 Sun et al.: Role of MiR-298 in Ischemia/Reperfusion Injury}

miR-298 inhibits the Act1/JNK/NF- $\kappa B$ pathway in vivo

To confirm the role of miR-298 in cerebral ischemic injury, we injected miR298 mimic into the right cerebral ventricle of mice, and miR-298 level in tissue samples collected from the cerebral hemisphere of the injured side after $1 \mathrm{~h}$ of MCAO and 12 h, 24 h, 48 h, 3 days, and 5 days after reperfusion was evaluated by qRT-PCR. miR-298 expression level was increased in MCAO samples (Fig. 10A). The results of the immunohistochemical analysis revealed that miR-298 mimic decreased the protein levels of pNF- $\kappa \mathrm{B}$ and pmTOR and increased those of pJNK, pBcl2, Beclin1, and Caspase- 3 in MCAO mice, which is similar to the trend observed in OGD/R N2a cells (Fig. 9). These results imply that miR-298 enhances autophagy.

miR-298 overexpression aggravates ischemic infarction and neurological deficits

An analysis of TTC staining revealed that ischemic injury was increased in the MCAO model as compared to sham mice (Fig. 10B, D). Injection of miR-298 mimic increased infarct volume in the brain 3 days after transient MCAO (Fig. 10B, D) and aggravated neurological deficits relative to control MCAO mice (Fig. 10C). The neurological score of each mouse in three groups was as follows: sham: $0,0,1,0,1,0$, 0, 1; I/R: 1, 2, 2, 2, 2, 3, 3, 2; miR-298: 4, 4, $4,3,3,2,4,4$. The observed downregulation of miR-298 in OGD/R cells and MCAO mice indicates that the change in miR-298 expression maybe attributed to ischemic injury.

\section{Discussion}

The finding of the study was that miR298 directly regulates the Act1/JNK/NF$\kappa \mathrm{B}$ pathway and inhibits the expression of Act1 protein in OGD/R-treated N2a cells and a mouse MCAO model of ischemic stroke. Moreover, miR-298 overexpression exacerbated neurological deficits in MCAO mice. Thus, high levels of miR-298 following ischemia/reperfusion can aggravate brain injury by suppressing Act1 protein expression.

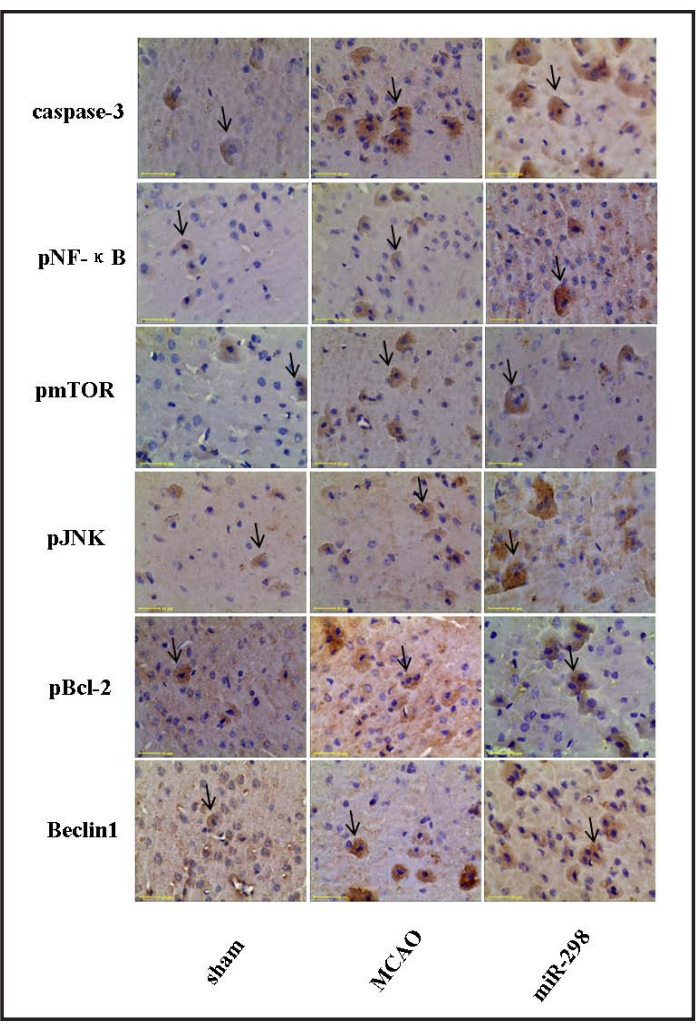

Fig. 9. Immunohistochemical analysis of Caspase-3, pNF- $\kappa$ B, pmTOR, pJNK, pBcl-2, and Beclin1 expression in MCAO model mice with or without miR-298 overexpression. The arrow indicated the cells with expressions of protein. $\mathrm{n}=8$ in each group.

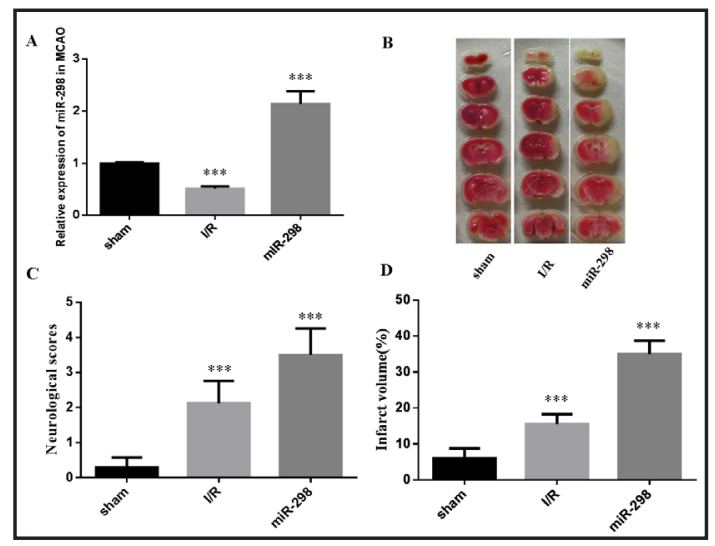

Fig. 10. MiR-298 mimic aggravated ischemic infarction and neurological dysfunction. A: Relative expression of miR-298 in MCAO model treated with miR-298 mimics. B: Evaluation of ischemic injury by TTC staining. C: neurological scores D: Infarct volume. $n=8$ in each group. Statistical method: oneway ANOVA; ${ }^{* * *} \mathrm{P}<0.001$ vs. sham group.

\section{KARGER}




\section{Cellular Physiology Cell Physiol Biochem 2018;48:528-539 \\ \begin{tabular}{c|c} 
DOI: 10.1159/000491810 & Ond 2018 The Author(s). Published by S. Karger AG, Basel \\
www.karger.com/cpb
\end{tabular}}

Sun et al.: Role of MiR-298 in Ischemia/Reperfusion Injury

Neuronal cell death is a critical aspect of stroke pathophysiology, and there is increasing evidence suggesting that miRNAs regulate this process, which includes apoptosis and autophagy [17]. For instance, brain-specific miR-124 suppresses the inhibitor of apoptosisstimulating protein of p53 levels and thereby enhances neuronal death [18]. Meanwhile, downregulation of miR-181b alleviated ischemia-induced neuronal death by blocking the translation of its target mRNA in vitro and in vivo [19]. In this study, we found that miR-298 overexpression increased both apoptosis and autophagy in vitro as well as ischemic infarct size in vivo, which can explain the observed impaired neurological function.

We previously showed that Act1 is a potential target of miR-298 based on the results of a bioinformatics analysis (RNAhybrid 2.2 database). In the present study, Act1 was upregulated in OGD/R N2a cells and MCAO mice. Given its ability to interact with interleukin-17 receptor and downstream components of the NF- $\kappa \mathrm{B}$ signaling pathway, Act1 is thought to function as an adaptor molecule linking these two elements [20]. Other studies have reported that Act1 activates JNK, and most ligands inducing NF- $\mathrm{kB}$ activation also activate mitogen-activated protein kinases and JNK [21-24]. The fact that Act1 activates both NF- $\kappa B$ and JNK indicates that it may act upstream of these factors. We observed increased levels of Act1 in OGD/R N2a cells and MCAO mice, and Act1 silencing decreased cell survival rate and increased apoptosis of OGD/R N2a cells. These data indicate that Act1 may play a protective role following ischemic stroke. Moreover, pJNK was upregulated and pNF- $\kappa B$ was downregulated by Act1 knockdown in OGD/R N2a cells. Together with our findings on cell survival and apoptosis, this suggests that Act1/JNK/NF- $\kappa B$ signaling is involved in brain injury following ischemic stroke.

miR-298 overexpression resulted in decreased Act 1 mRNA and protein levels. MiRNAs bind to the 3' UTR of target transcripts to induce mRNA degradation or inhibit protein translation. We confirmed that Act1 is a direct target of miR-298 with the luciferase assay. Our data show that miR-298 negatively regulates Act1 expression in ischemic stroke.

Ischemia/reperfusion injury is associated with neuronal damage, while hypoxia is a known inducer of autophagy [25]. The relationship between apoptosis and autophagy has been investigated in many recent studies [26]. The upregulation of Beclin 1 in the present study suggests that miR-298 overexpression enhances cell autophagy following ischemic stroke. We investigated the signaling pathways involved by examining the expression and

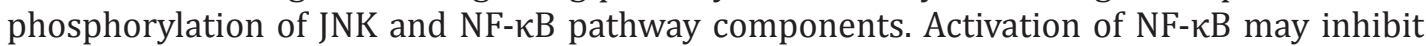
autophagy following cerebral ischemia through regulation of mTOR signaling; thus, blocking autophagy by increasing NF- $\kappa B$ levels is a potential strategy for the treatment of ischemic stroke [27]. Since Act1 is an activator of NF- $\kappa B$ [28], its knockdown may lead to downregulation of NF-kB. In the present study, miR-298 overexpression decreased the protein levels of Act1, pNF- $\kappa B$, and pmTOR, an effect that was reversed by overexpressing Act1. These data indicate that the miR-298/Act1/NF- $\kappa$ B cascade may regulate autophagy in ischemic stroke. Regulation of the JNK/Bcl-2/Beclin1 signaling may underlie OGD/R-induced autophagy in neurons and the consequent decrease in neuronal viability [29]. This is associated with JNK activation, Bcl-2 phosphorylation and inactivation, and the dissociation of the Bcl-2/Beclin-1 complex. In our study, overexpression of miR-298 decreased Act1 and increased pJNK, pBcl2, and Beclin1 levels; the levels were restored by overexpression of Act1. Together with our observation Act1 knockdown leads to an increase in JNK phosphorylation, we propose that the miR-298/Act1/JNK regulates neuronal autophagy in ischemic stroke.

\section{Conclusion}

The results of the study show that miR-298 overexpression aggravates ischemic injury in vitro and in vivo through negative regulation of Act1/JNK/NF- $\mathrm{KB}$ and downstream autophagy pathways. These findings suggest potential therapeutic targets for the treatment of ischemic stroke, although a more detailed investigation of the molecular mechanisms using a transgenic animal model is warranted. 


\section{Cellular Physiology Cell Physiol Biochem 2018;48:528-539

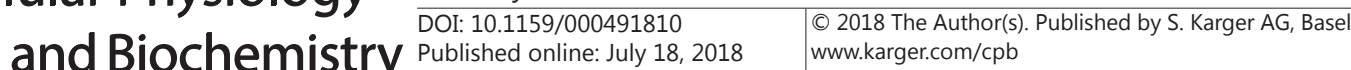

Sun et al.: Role of MiR-298 in Ischemia/Reperfusion Injury

\section{Acknowledgements}

This work was supported by the National Natural Science Foundation of China (grant no. 81471204), and the Graduate Innovative Research Projects of Harbin Medical University (grant no. YJSCX2017-28HYD).

\section{Disclosure Statement}

The authors declare that they have no competing interests.

\section{References}

1 Yue YH, Bai XD, Zhang HJ, Li YM, Hu L, Liu LY, Mao JP, Yang XY, Dila NM: Gene Polymorphisms Affect the Effectiveness of Atorvastatin in Treating Ischemic Stroke Patients. Cell Physiol Biochem 2016;39:630-638.

-2 Higashida RT, Furlan AJ, Roberts H, Tomsick T, Connors B, Barr J, Dillon W, Warach S, Broderick J, Tilley B, Sacks D: Trial design and reporting standards for intra-arterial cerebral thrombolysis for acute ischemic stroke. Stroke 2003;34:109-137.

-3 Ouyang YB, Giffard RG: ER-Mitochondria Crosstalk during Cerebral Ischemia: Molecular Chaperones and ER-Mitochondrial Calcium Transfer. Int J Cell Biol 2012;2012:493934.

4 Kodaira H, Kusuhara H, Fujita T, Ushiki J, Fuse E, Sugiyama Y: Quantitative evaluation of the impact of active efflux by p-glycoprotein and breast cancer resistance protein at the blood-brain barrier on the predictability of the unbound concentrations of drugs in the brain using cerebrospinal fluid concentration as a surrogate. J Pharmacol Exp Ther 2011;339:935-944.

-5 Li X, Commane M, Nie H, Hua X, Chatterjee-Kishore M, Wald D, Haag M, Stark GR: Act1, an NF-kappa B-activating protein. Proc Natl Acad Sci USA 2000;97:10489-10493.

6 Leonardi A, Chariot A, Claudio E, Cunningham K, Siebenlist U: CIKS, a connection to Ikappa B kinase and stress-activated protein kinase. Proc Natl Acad Sci USA 2000;97:10494-10499.

7 Liu C, Qian W, Qian Y, Giltiay NV, Lu Y, Swaidani S, Misra S, Deng L, Chen ZJ, Li X. Act1, a U-box E3 ubiquitin ligase for IL-17 signaling. Sci Signal 2009;2:ra63.

8 Yu D, Yang L, Feng Y, Fan C, Wang S, Mo X: MicroRNAs expression and function in cerebral ischemia reperfusion injury. J Mol Neurosci 2014;53:242-250.

-9 Zhao H, Zhao D, Tan G, Liu Y, Zhuang L, Liu T: Bufalin promotes apoptosis of gastric cancer by downregulation of miR-298 targeting bax. Int J Clin Exp Med 2015;8:3420-3428.

10 Zhou F, Chen J, Wang H: MicroRNA-298 inhibits malignant phenotypes of epithelial ovarian cancer by regulating the expression of EZH2.Oncol Lett 2016;12:3926-3932.

11 Bao L, Hazari S, Mehra S, Kaushal D, Moroz K, Dash S: Increased expression of P-glycoprotein and doxorubicin chemoresistance of metastatic breast cancer is regulated by miR-298.Am J Pathol 2012;180:2490-2503.

12 Boissonneault V, Plante I, Rivest S, Provost P: MicroRNA-298 and microRNA-328 regulate expression of mouse beta-amyloid precursor protein-converting enzyme 1. J Biol Chem 2009;284:1971-1981.

13 Yao Y, Chen L, Xiao J, Wang C, Jiang W, Zhang R, Hao J. Chrysin protects against focal cerebral ischemia/ reperfusion injury in mice through attenuation of oxidative stress and inflammation. Int J Mol Sci 2014;15:20913-20926.

14 Liu DZ, Tian Y, Ander BP, Xu H, Stamova BS, Zhan X, Turner RJ, Jickling G, Sharp FR. Brain and blood microRNA expression profiling of ischemic stroke, intracerebral hemorrhage, and kainate seizures. J Cereb Blood Flow Metab 2010;30:92-101.

15 Longa EZ, Weinstein PR, Carlson S, Cummins R. Reversible middle cerebral artery occlusion without craniectomy in rats. Stroke 1989; 20:84-91.

16 Pei L, Meng S, Yu W, Wang Q Song F, Ma L. Inhibition of MicroRNA-383 Ameliorates Injury After Focal Cerebral Ischemia via Targeting PPAR $\gamma$. Cell Physiol Biochem 2016;39:1339-1346.

17 Baczyńska D, Michałowska D, Witkiewicz W: The role of microRNA in ischemic diseases--impact on the regulation of inflammatory, apoptosis and angiogenesis processes. Przegl Lek 2013;70:135-142. 


\section{Cellular Physiology Cell Physiol Biochem 2018;48:528-539 \begin{tabular}{l|l} 
DOI: 10.1159/000491810 & $\begin{array}{l}\text { O 2018 The Author(s). Published by S. Karger AG, Basel } \\
\text { www.karger.com/cpb }\end{array}$
\end{tabular} \\ Sun et al.: Role of MiR-298 in Ischemia/Reperfusion Injury}

18 Liu X, Li F, Zhao S, Luo Y, Kang J, Zhao H, Yan F, Li S, Ji X: MicroRNA-124-mediated regulation of inhibitory member of apoptosis-stimulating protein of p53 family in experimental stroke. Stroke 2013;44:1973-1980.

19 Peng Z, Li J, Li Y, Yang X, Feng S, Han S, Li J: Downregulation of miR-181b in mouse brain following ischemic stroke induces neuroprotection against ischemic injury through targeting heat shock protein A5 and ubiquitin carboxyl-terminal hydrolase isozyme L1. J Neurosci Res 2013;91:1349-1362.

20 Kanamori M, Kai C, Hayashizaki Y, Suzuki H: NF-kappaB activator Act1 associates with IL-1/Toll pathway adaptor molecule TRAF6. FEBS Lett. 2002;532:241-246.

-21 Valente AJ, Yoshida T, Clark RA, Delafontaine P, Siebenlist U, Chandrasekar B: Advanced oxidation protein products induce cardiomyocyte death via Nox2/Rac1/superoxide-dependent TRAF3IP2/JNK signaling. Free Radic Biol Med 2013;60:125-135.

-22 Li W, Fan M, Chen Y, Zhao Q Song C, Yan Y, Jin Y, Huang Z, Lin C, Wu J: Melatonin Induces Cell Apoptosis in AGS Cells Through the Activation of JNK and P38 MAPK and the Suppression of Nuclear Factor-Kappa B: a Novel Therapeutic Implication for Gastric Cancer. Cell Physiol Biochem 2015;37:2323-2338.

23 Lee SY, Reichlin A, Santana A, Sokol KA, Nussenzweig MC, Choi Y: TRAF2 is essential for JNK but not NFkappaB activation and regulates lymphocyte proliferation and survival. Immunity 1997;7:703-713.

24 Jia XJ, Li X, Wang F, Liu HQ, Zhang DJ, Chen Y: Berbamine Exerts Anti-Inflammatory Effects via Inhibition of NF-кB and MAPK Signaling Pathways. Cell Physiol Biochem. 2017;41:2307-2318.

25 Wu J, Niu J, Li X, Li Y, Wang X, Lin J, Zhang F: Hypoxia induces autophagy of bone marrow-derived mesenchymal stem cells via activation of ERK1/2. Cell Physiol Biochem 2014;33:1467-1474.

-26 Wang XM, Yang YJ, Wu YJ, Zhang Q, Qian HY: Attenuating Hypoxia-Induced Apoptosis and Autophagy of Mesenchymal Stem Cells: the Potential of Sitagliptin in Stem Cell-Based Therapy. Cell Physiol Biochem 2015;37:1914-1926.

27 Li WL, Yu SP, Chen D, Yu SS, Jiang YJ, Genetta T, Wei L: The regulatory role of NF- $\kappa$ B in autophagy-like cell death after focal cerebral ischemia in mice. Neuroscience 2013;244:16-30.

28 Dong Z, Yang Y, Zhang T, Li Y, Kang Q, Lei W, Cao Y, Niu X, Wang D, Tai W: siRNA-Act1 inhibits the function of IL-17 on lung fibroblasts via the NF-кB pathway. Respiration 2013;86:332-340.

29 Fan J, Liu Y, Yin J, Li Q, Li Y, Gu J, Cai W, Yin G: Oxygen-Glucose-Deprivation/Reoxygenation-Induced Autophagic Cell Death Depends on JNK-Mediated Phosphorylation of Bcl-2. Cell Physiol Biochem 2016;38:1063-1074. 